PROCEEDINGS OF THE

AMERICAN MATHEMATICAL SOCIETY

Volume 128, Number 2, Pages 383-386

S 0002-9939(99)05005-4

Article electronically published on July 6, 1999

\title{
THE SUM OF TWO LOCALLY NILPOTENT RINGS MAY CONTAIN A FREE NON-COMMUTATIVE SUBRING
}

\author{
ANNA FUKSHANSKY
}

(Communicated by Ken Goodearl)

\begin{abstract}
A family of examples of semigroup algebras is constructed each of which is a sum of two locally nilpotent subalgebras but yet contains a free subalgebra which is freely generated by two elements.
\end{abstract}

In this paper we consider rings which are sums of two subrings. Kegel [1] showed that a ring which is the sum of two nilpotent subrings is nilpotent. Kelarev showed in [2] that a ring which is the sum of two locally nilpotent subrings might not be nil. In the papers [4] and [3] we find some other constructions of rings, that are sums of locally nilpotent subrings, but which are not nil. We use the construction described in [4], [3] to give an example of an associative algebra which is the sum of two locally nilpotent subalgebras, but which contains a free subalgebra. The two subalgebras are even more than locally nilpotent: Every finitely generated ideal in the subalgebras is nilpotent.

We want to remind the reader of some definitions about semigroups:

Definition. 1. If $I$ is an ideal of the semigroup $S$, then the contraction $S_{I}:=$ $S \backslash I \cup\{0\}$ is again a semigroup, where 0 is a possibly new element and is zero in $S_{I}$

2. Let $K$ be a field and $S$ a semigroup.

$$
\mathbf{K S}:=\left\{\sum_{a \in S^{\#}} k_{a} a \mid k_{a} \in K, \text { all but finitely many } k_{a} \text { are zero }\right\}
$$

is the set of linear combinations over $K$, where $S^{\#}$ is $S$ without zero. $K S$ is called the semigroup algebra over $K$.

Note that the usual definition of the semigroup algebra allows the zero of the semigroup (if it exists) to be an element of the basis - in this paper however we will use the above definition. Sometimes a semigroup can be written as a disjoint union of subsemigroups, as e.g. the natural numbers with the usual multiplication are the union of odd and even numbers. The next lemma shows what we can say about the semigroup algebra in this case.

Received by the editors October 9, 1997 and, in revised form, April 9, 1998.

1991 Mathematics Subject Classification. Primary 16N40; Secondary 20M25.

Key words and phrases. Nilpotent rings, locally nilpotent rings, nil rings.

(C)1999 American Mathematical Society 
Lemma. Let $A$ be a semigroup, and $A_{1}$ and $A_{2}$ subsemigroups of $A$, such that $A=A_{1} \cup A_{2}$ and $A_{1} \cap A_{2} \subseteq\{0\}$. Then $K A=K A_{1} \oplus K A_{2}$, i.e. a direct sum of the two vector subspaces which are subalgebras.

Now we can formulate our

Theorem. There exists an associative algebra $A$, which is a direct sum of two subalgebras $A_{1}, A_{2}$, where $A_{1}$ and $A_{2}$ are locally nilpotent, but which contains a free subalgebra.

To prove the theorem we have to construct such an algebra, which will be a semigroup algebra. The subalgebras of the constructed example will have the following property: Every finite subset generates a nilpotent ideal, so there is a stronger form of nilpotency in these subalgebras.

Given an associative algebra $A$ one always obtains a Lie algebra $A^{L}$ which has the same additive group and the Lie multiplication

$$
[a, b]:=a b-b a \text { for every } a, b \in A^{L} .
$$

If the algebra $A$ is nilpotent, locally nilpotent, or if it contains a free associative subalgebra the Lie algebra $A^{L}$ will be nilpotent, locally nilpotent, or it will contain a free Lie subalgebra. We deduce the following

Corollary. There exists a Lie algebra L, which is a direct sum of two subalgebras $L_{1}, L_{2}$, where $L_{1}$ and $L_{2}$ are locally nilpotent, but $L$ contains a free Lie subalgebra.

Proof of the theorem. Let $S$ be the free semigroup (without 1) generated by the letters $a$ and $b$. We define a function from $S$ into the real numbers

$$
\begin{aligned}
& d: S \longrightarrow \mathbf{R} \\
& w \longmapsto d(w):=t n_{b}-n_{a}
\end{aligned}
$$

where $n_{b}$ and $n_{a}$ denote the number of $b$ 's and $a$ 's in the word $w$, respectively, and $t$ is a irrational number with $1<t<2$ (and guarantees that $d(w) \neq 0$ for every $w \in S)$. Define subsets of $S$ :

$$
A:=\{s \in S \mid d(s)<0\} \quad \text { and } \quad B:=\{s \in S \mid d(s)>0\} .
$$

Since $d(w) \neq 0$ for every $w \in S, S$ is the disjoint union of $A$ and $B$. Using the additivity of the function $d$, i.e. $d\left(s \cdot s^{\prime}\right)=d(s)+d\left(s^{\prime}\right)$, we see that $A$ and $B$ are subsemigroups of $S$. Denote by $I$ the ideal of $S$ generated by the words satisfying $|d(s)| \geq 8 t:$

$$
I:=\langle\{s \in S|:| d(s) \mid \geq 8 t\}\rangle_{\text {ideal }},
$$

and define a right ideal of $S, R I$, to be generated as follows:

$$
R I:=\langle\{s \in S|:| d(s) \mid \geq 4 t\}\rangle_{\text {right ideal }} .
$$

Since $b^{8} \in I$ and $b \notin I$ we have $\emptyset \neq I \neq S$. The contraction $S_{I}$ is still the union of $A_{I \cap A}$ and $B_{I \cap B}$. We will write this as:

$$
S_{I}=A_{I} \cup B_{I},
$$

note that $A_{I} \cap B_{I}=\{0\}$. Hence choosing any field $K$ we get $K S_{I}=K A_{I} \oplus K B_{I}$. The algebra $K S_{I}$ depends on $t$, for every choice of $t \in\{\xi \in \mathbb{R} \backslash \mathbb{Q} \mid 2>\xi>1\}$ it is an example proving our theorem.

In the same way as in [3] (where $t=\sqrt{2}$ and $I=\langle\{s \in S|:| d(s) \mid \geq 3\}\rangle$ ) one shows that $K A_{I}$ and $K B_{I}$ are locally nilpotent and even that every finitely generated ideal of $K A_{I}$ and $K B_{I}$ is nilpotent. 
Claim. The elements $x=a a a+b b b$ and $y=a b a+b a b$ generate a free subalgebra in $K S_{I}$.

To prove this we will have to show that no non-trivial linear combination of products in $x$ and $y$ is zero. We will do this in four steps.

1. The elements $x$ and $y$ are linearly independent.

Let $k x+l y=0$ for some $l, k \in K$. Then $k a a a+k b b b+l a b a+l b a b=0$. But $a a a, b b b, a b a, b a b$ are in $S_{I}^{\#}$ which is a basis for $K S_{I}$ and therefore $k=l=0$.

2. The elements $x$ and $y$ are not nilpotent.

Consider $x$ first. We show by induction on $n$ :

$x^{n}$ has a summand, which we shall call 'good summand', which lies in $S \backslash R I$ and $|d(s)|<3 t$.

If $n=1$, then both $a a a$ and $b b b$ are good summands. Now let $n \geq 1, x^{n+1}=x^{n} x$, and $x^{n}$ has a good summand, say $s$ :

$$
x^{n+1}=(\ldots+s+\ldots)(a a a+b b b)=s a a a+s b b b+\ldots \quad .
$$

If $d(s)>0$, then saaa is a good summand, if $d(s)<0$, then $s b b b$ is a good summand.

To show that $y$ is not nilpotent, we proceed in the same way. We prove by induction on $n$ :

$y^{n}$ has a summand $s$, which we shall call 'good summand', which lies in $S \backslash R I$ and $|d(s)|<3 t$.

If $n=1$, then both $a b a$ and $b a b$ are good summands. Let $n \geq 1, y^{n+1}=y^{n} y$, and $y^{n}$ has a good summand, say $s$ :

$$
y^{n+1}=(\ldots+s+\ldots)(a b a+b a b)=s a b a+s b a b+\ldots \quad .
$$

Again if $d(s)>0$, then saba is a good summand, if $d(s)<0$, then $s b a b$ is a good summand.

Remark. (1) The bound $b_{I}$ on the $d$-value of the generating elements of the ideal $I$ must be at least twice as big as the bound $b_{R I}$ concerning the right ideal $R I$ to make sure that $R I \supseteq I$ (here $b_{I}=8 t, b_{R I}=4 t$ ). The bound $b_{g s}$ which defines the good summand (here $b_{g s}=3 t$ ) must satisfy $b_{g s}+t \leq b_{R I}$ to make sure that $y$ is not nilpotent. The chosen bounds $3 t, 4 t$, and $8 t$ are the smallest such that $x$ and $y$ each have two good summands.

(2) Since our ideal is generated by the words $s$ such that $|d(s)| \geq 8 t$ we prove at the same time that e.g. $\tilde{y}=b b a+a a b$ is not nilpotent.

(3) $S_{I}$ is infinite.

3. A product $\pi$ in $x$ and $y$ is never zero.

We prove this by induction on the length of the product $\pi$ and use the same idea as in step 2.

We want to prove: For any $n$ the product $\pi$ of length $n$ in $x$ and $y$ has a summand $s$, which we shall call a 'good summand', which lies in $S \backslash R I$ and $|d(s)|<3 t$.

If $n=1$ this is true, for $\pi$ is just $x$ or $y$.

Let $\pi=z_{1} \cdots z_{n} z_{n+1}$ and let $z_{1} \cdots z_{n}$ have a good summand, say $s$. So $\pi=$ $(\ldots+s+\ldots) z_{n+1}$. Depending on $d(s)$ being greater or smaller than zero and on $z_{n+1}$ being $x$ or $y$ we find one of saaa, sbbb, saba, sbab to be a good summand of $\pi$.

4. No non-trivial linear combination of words in $x$ and $y$ is zero. A linear combination $\omega$ looks like this:

$$
\omega=a_{1} s_{1}+\cdots+a_{m} s_{m}, \quad 0 \neq a_{1}, \ldots, a_{m} \in K,
$$


where each $s_{i}$ is a product in $x, y$ and we may replace $s_{i}$ by a sum of products in $a, b$. Let us call these products $s(a, b)_{i, j}$, where $j$ goes from 1 to the number of non-zero such products which come from $s_{i}$. Note, that because of the choice of $x$ and $y$ each $s(a, b)_{i, j}$ is three times as long as $s_{i}$ and it is easy to see that $s(a, b)_{i, j} \neq s(a, b)_{i, k}$ for $j \neq k$.

Suppose $\omega=0$. Then there must exist $i, k \in \mathbb{N}, i \neq 1$, such that the first nonzero summand of $\pi_{1}$ must occur as a non-zero summand of some other $\pi_{j}$, i.e. $s(a, b)_{1,1}=s(a, b)_{j, k}$. Since $\pi_{1} \neq \pi_{i}$ they differ in at least one $(x, y)$-letter. Say for some $l \leq n, z_{k}, z_{k}^{\prime} \in\{x, y\}, l \leq n$,

$$
\pi_{1}=z_{1} \cdots z_{l-1} x z_{l+1} \cdots z_{n} \text { and } \pi_{j}=z_{1} \cdots z_{l-1} y z_{l+1}^{\prime} \cdots z_{n}^{\prime} .
$$

But then any $s(a, b)_{j, k}$ has the pattern $a b a$ or $b a b$ at the places $3 l+1,3 l+2,3 l+3$, and $s(a, b)_{1,1}$ must have at these places the pattern $a a a$ or $b b b$ inherited from the letter $x$. So they never can be equal.

Thus this linear combination with different $s_{i}$ 's can never be zero. We have proved that the elements $x$ and $y$ generate a free subalgebra of $K S_{I}$.

\section{REFERENCES}

[1] O.H. Kegel, 'Zur Nilpotenz gewisser assoziativer Ringe', Math. Ann. 149(1962/63), 258-260. MR 28:3049

[2] A.V. Kelarev, 'A sum of two locally nilpotent rings may be not nil', Arch. Math. 60(1993), 431-435. MR 94c: 16025

[3] A.V. Kelarev, 'A primitive ring which is a sum of two Wedderburn radical subrings', Proc. Amer. Math. Soc. 125 (1997), No.7, pp 2191-2193. MR 97i:16004

[4] A. Salwa, 'Rings that are sums of two locally nilpotent subrings', Comm. Algebra 24(12)(1996), 3921-3931. MR 97e:16051

Martin-Luther-Universität Halle-Wittenberg, Fachbereich Mathematik und Informatik, Institut für Algebra und Geometrie, 06099 Halle, Germany

Current address: Department of Computer Science, Royal Holloway University of London, Egham Surrey TW20 0EX, United Kingdom

E-mail address: A.Fukshansky@dcs.rhbnc.ac.uk 